\title{
Saposin-lipoprotein scaffolds for structure determination of membrane transporters
}

Joseph Lyons ${ }^{1}$, Andreas Bøggild ${ }^{1}$, Poul Nissen ${ }^{1}$, Jens Frauenfeld ${ }^{2}$

${ }^{1}$ Aarhus University, Department of Molecular Biology and Genetics, 8000 Aarhus C, Denmark

${ }^{2}$ Salipro Biotech AB, Teknikringen 38a, 11428 Stockholm, Sweden

\section{Contents}

Introduction 4

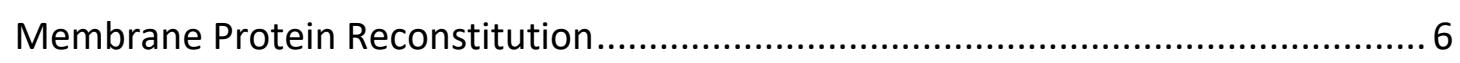

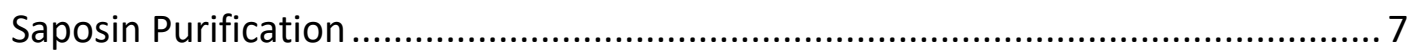

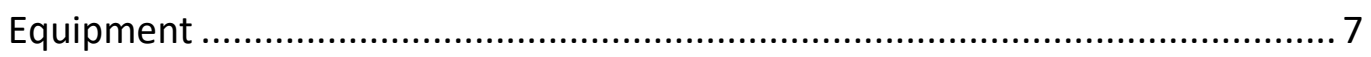

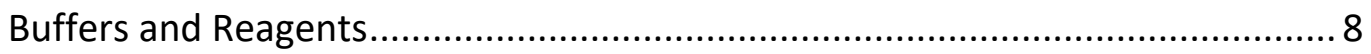

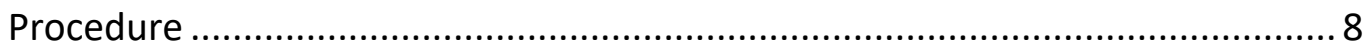

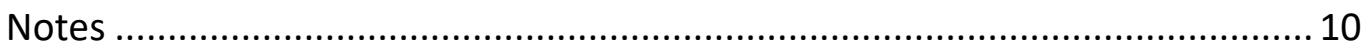

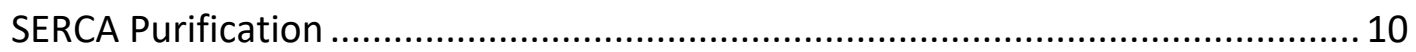

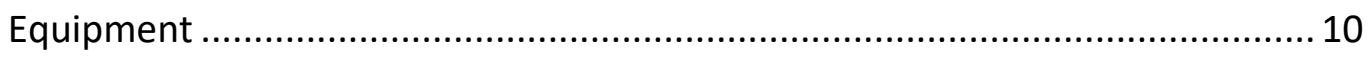

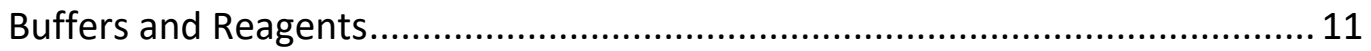

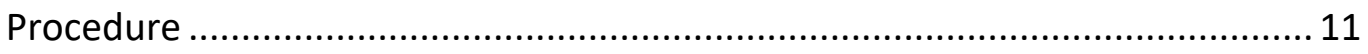

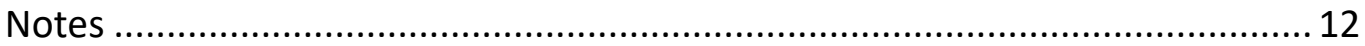

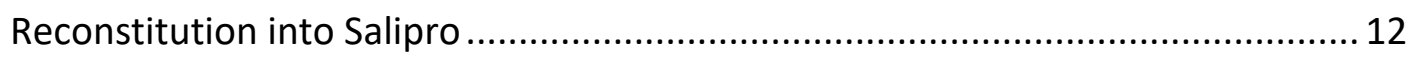

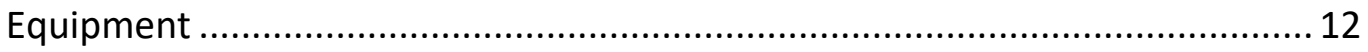

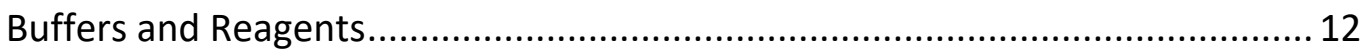

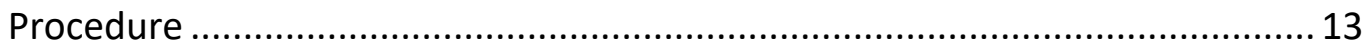


Notes

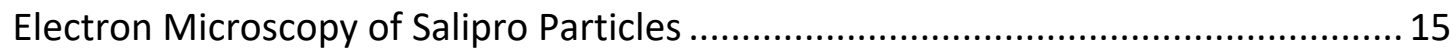

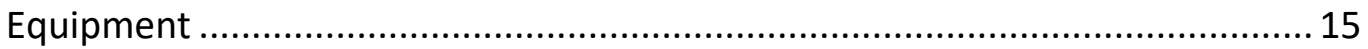

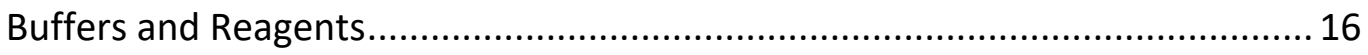

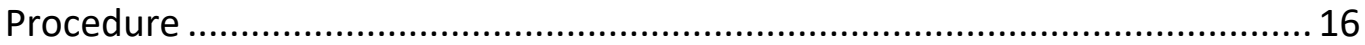

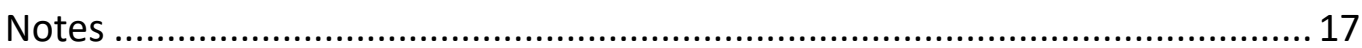

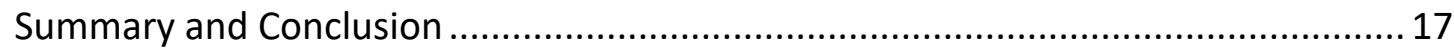

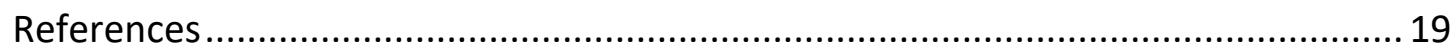

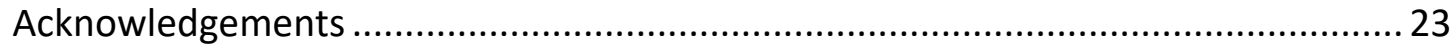

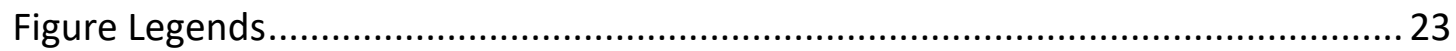

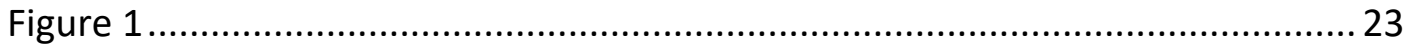

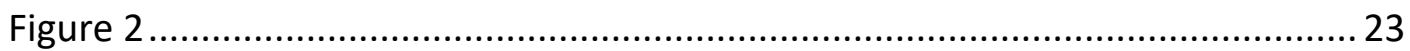

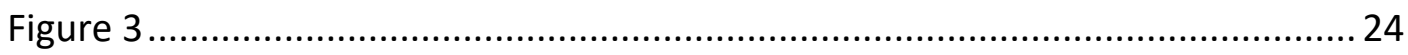

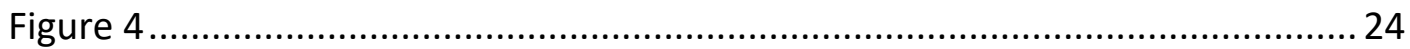

\section{Abstract}

Membrane proteins depend on their natural lipid environment for function, which makes them more difficult to study in isolation. A number of approaches that mimic the lipid bilayer of biological membranes have been described (nanodiscs, SMALPs), enabling novel ways to assay activity and elucidate structures of this important class of proteins. More recently, the use of saposin A, a protein that is involved in lipid transport, to form Salipro (saposin-lipid-protein) complexes was demonstrated for a range of targets (Frauenfeld et al., 2016). The method is fast and requires little resources. The saposin-lipid-scaffold adapts to various sizes of transmembrane 
regions during self-assembly, forming a minimal lipid nanoparticle. This results in the formation of a well-defined membrane protein-lipid complex, which is desirable for structural characterization.

Here we describe a protocol to reconstitute the Sarco-Endoplasmic Reticulum Calcium ATPase (SERCA) into Salipro lipid nanoparticles. The complex formation is analyzed using negative stain EM, allowing to quickly determine an initial structure of the membrane protein and to evaluate sample conditions for structural studies using single particle cryo-EM in a detergent-free environment. 


\section{Introduction}

Membrane proteins are among the most challenging proteins for structural characterization, as they are not readily purified into solution, but instead require detergents to solubilize them from their native lipid bilayer. For crystallization, the typical approach is either to attempt crystallization in detergent micelles, add lipids for crystallization in a bilayer-like environment (Faham \& Bowie, 2002; Gourdon et al., 2011; Poulos, Morgan, Zimmer, \& Faham, 2015), or crystallize in lipidic cubic phase (Caffrey, 2015).

Several methods have been proposed to stabilize membrane proteins in a more natural lipid environment, while keeping them in solution. One such method is to incorporate the target membrane protein into nanodiscs (Bayburt, Grinkova, \& Sligar, 2002), which are small sections of a lipid bilayer, encircled by MSPs (Membrane Scaffold Proteins). The dimension of the MSP based nanodisc and its ability to incorporate membrane proteins of various sizes is dictated by exploiting MSPs of differing lengths, with reconstitution success often requiring extensive optimization for a given protein target. While successes have been reported for single particle cryo-electron microscopy (SP cryo-EM) studies of nanodisc reconstituted membrane proteins, these have been limited to larger membrane protein targets (Baker, Fan, \& Serysheva, 2015; Frauenfeld et al., 2011; Gao, Cao, Julius, \& Cheng, 2016).

A more recent alternative has been reported, which uses styrene maleic acid copolymers (SMAs) to generate SMALPs (SMA Lipid Particles), which allows for the isolation of membrane proteins directly from its native membrane, without the need 
for detergents (Jamshad et al., 2011). Negative stain analysis of AcrB reconstituted into SMALPs has provided an initial proof of concept for the use of SMALPs in structural studies (Postis et al., 2015).

Another new technological breakthrough, and the focus of this Chapter, exploits the self-assembly of lipid and saposin into a saposin-lipoprotein nanoparticle (Salipro). The saposin protein family comprises four (saposin A-D) small proteins ( $10 \mathrm{kDa}$ ) that derive from a precursor proteins involved as co-factors in the catabolism of sphingolipids. Crystal structures of saposin proteins have been reported with and without bound detergent/lipid (Ahn, Leyko, Alattia, Chen, \& Privé, 2006; Popovic, Holyoake, Pomès, \& Privé, 2012). Frauenfeld et al. rationalized that these proteins could be exploited as scaffold proteins to reconstitute membrane proteins in a lipid environment and reported on its application to a number of membrane proteins. Saposin A can be expressed and purified from Escherichia coli. Its general versatility lies in its ability to adapt the size of the nanoparticle to the membrane protein, adjusting to transmembrane regions of varying size (Frauenfeld et al., 2016). This reduces the need to screen for a variety of scaffold protein constructs with varying protein to lipid ratios and thereby reduces the complexity of the reconstitution. The experimental setup is rather straightforward, where in a rapid process the saposin scaffold, lipids and membrane proteins self-assemble into robust lipid nanoparticles. To date, mainly the human saposin A has been exploited to generate Salipro particles, however other saposins from the saposin protein family might provide additional versatility for specific uses, such as in combination with certain lipids or classes of membrane proteins. 
Electron microscopy has seen rapid advances over the last few years, in what has been called the "resolution revolution" (Kühlbrandt, 2014). For instance, new detectors allow for high-resolution data $(\sim 2 \AA)$ to be collected, while the use of phase plates provide a boost to contrast, allowing smaller particles to be identified in the micrographs (Danev \& Baumeister, 2016). These advances coupled with the improvements to sample supports, microscopes, new software and computer hardware makes working with large cryo-EM datasets easier and allows for the visualization and atomic model building of membrane proteins.

Here we demonstrate that Salipro reconstitution can be used for structural characterization of P-type ATPases, using SERCA1a natively isolated from the fasttwitch muscle of rabbit as a test case. We believe the approach described here can easily be applied to other transporters, channels and receptors.

\section{Membrane Protein Reconstitution}

For the reconstitution into Salipro particles, membrane proteins may originate either from natural sources or from overexpression in appropriate expression systems, e.g., bacterial, yeast or mammalian system (Lyons, Shahsavar, Paulsen, Pedersen, \& Nissen, 2016). Membrane proteins require solubilization in an appropriate detergent, followed by one or more purification steps. The choice of detergent is often critical as some detergents might severely reduce the stability of the protein. As this is generally established on a case-to-case basis, we will not describe these initial steps here (for review, see (Loll, 2014)), but instead start with the final purification step before Salipro reconstitution. 
Once the sample of pure solubilized membrane protein is available, reconstitution into Salipro can proceed quite straightforwardly, with purification by a final size exclusion chromatography step to separate the reconstituted membrane proteinSalipro particle from free saposin, lipid-only (empty) Salipro particles and potential aggregates. However, while the reconstitution protocol is fast, it will likely require some screening of the various parameters to find the optimal strategy for reconstitution. The choice of lipid(s) to use could be one such parameter, along with incubation times and temperature, and ratios between the target protein, saposin and lipid(s).

\section{Saposin Purification}

Saposin proteins are capable of binding lipids. This class of proteins is composed of amphipathic helices with hydrophobic amino acids that build the protein core, and six invariable cysteine residues that form disulphide bridges that lead to an extraordinary stability of the domains.

\section{Equipment}

- Baffled conical flasks

- ÄKTA chromatography system

- HiLoad Superdex 200 16/600 GL

- Centrifuges for the harvesting of $\mathrm{E}$. coli cells (at least $6,000 \times \mathrm{g}$ ) and $\mathrm{a}$ standard refrigerated centrifuge capable of speeds of up to $26,000 \times \mathrm{g}$

- Probe sonicator suitable for lysing E. coli.

- Water bath

- 10-mm-(i.d.) open gravity flow column 


\section{Buffers and Reagents}

- E. coli Rosetta gami-2 (DE3) (Novagen)

- Terrific Broth (TB) medium

- Tetracycline, Chloramphenicol, Kanamycin

- IMAC resin: Ni Sepharose 6 Fast Flow

- Lysis buffer: 20 mM HEPES pH 7.5, 150 mM NaCl, 20 mM Imidazole

- Wash buffer: 20 mM HEPES pH 7.5, 150 mM NaCl, 40 mM Imidazole

- Elution buffer: 20 mM HEPES pH 7.5, 150 mM NaCl, 400 mM Imidazole

- Size Exclusion buffer: 20 mM HEPES pH 7.5, $150 \mathrm{mM} \mathrm{NaCl}$

- TEV protease containing uncleavable His-tag

- Amicon Ultra concentrator (3000 Da MWCO)

- Dialysis bag (1000 Da MWCO)

\section{Procedure}

1. Saposin A is expressed using a vector with the coding region for saposin A inserted into a pNIC-Bsa4 plasmid. This plasmid adds an N-terminal hexahistidine tag with an integrated TEV protease cleavage site.

2. Cultures of $1 \mathrm{~L}$ Terrific Broth (TB) medium supplemented with Tetracycline, Chloramphenicol and Kanamycin in $2.5 \mathrm{~L}$ baffled conical flasks are inoculated from an overnight culture and grown at $37^{\circ} \mathrm{C}$ at $200 \mathrm{rpm}$.

3. Protein expression is induced at OD $600 \mathrm{~nm}$ of 1.0 with $0.7 \mathrm{mM}$ IPTG at $37^{\circ} \mathrm{C}$. Three hours after induction, cells are harvested at 12,000xg for 20 minutes. At this point, cells can be frozen for long-term storage in a $-80^{\circ} \mathrm{C}$ freezer. 
4. For purification, cells are resuspended in Lysis buffer ( $1 \mathrm{~g}$ of cell wet weight in $5 \mathrm{ml}$ lysis buffer) and disrupted by sonication.

5. Cell debris is removed by centrifugation at $26,000 \times \mathrm{g}$ for $30 \mathrm{~min}$ at $4^{\circ} \mathrm{C}$.

6. In a waterbath, the supernatant is incubated at $85^{\circ} \mathrm{C}$ for $10 \mathrm{~min}$.

7. The supernatant is subjected to a second centrifugation step at $26,000 \times \mathrm{g}$ for $30 \min$ at $4^{\circ} \mathrm{C}$.

8. IMAC resin (Ni Sepharose 6 Fast Flow) is equilibrated with Lysis buffer (10 CV) in 10-mm-(i.d.) open gravity flow columns.

9. Transfer of IMAC resin to a falcon tube and incubated with lysate $(1 \mathrm{ml}$ of resin for $20 \mathrm{ml}$ lysate) for $30 \mathrm{~min}$ at $4{ }^{\circ} \mathrm{C}$.

10. After binding of saposin A to the IMAC resin, the chromatography medium is packed in open gravity flow columns.

11. Unbound proteins are removed by washing with 15 CV of Lysis buffer, followed by $15 \mathrm{CV}$ of Wash buffer.

12. Saposin A is eluted by adding $5 \mathrm{CV}$ of Elution buffer.

13. Saposin A is subsequently dialyzed against Size Exclusion buffer together with TEV protease overnight in a dialysis bag at $4{ }^{\circ} \mathrm{C}$ with gentle stirring.

14. To remove TEV protease and uncleaved proteins, the sample is passed over equilibrated IMAC beads again and the flow through collected and concentrated using Amicon Ultra concentrators to a volume of $5 \mathrm{ml}$.

15. Using an ÄKTA chromatography system, the sample is loaded onto a HiLoad Superdex $20016 / 600 \mathrm{GL}$ column, equilibrated with at least two column volumes of Size Exclusion buffer, and fractions are collected. 
16. Peak fractions are pooled and concentrated to $1.2 \mathrm{mg} / \mathrm{ml}$ protein using an Amicon Ultra concentrator.

17. Aliquot and flash freeze purified saposin $\mathrm{A}$ in liquid nitrogen for storage at -80 ${ }^{\circ} \mathrm{C}$.

Notes

1. Typically, a 1-liter bacterial culture yields 5-10 mg of purified saposin.

\section{SERCA Purification}

For the purpose of this protocol, it is assumed that one has a purified membrane protein, therefore details pertaining to the expression and purification strategies will not be discussed in detail here. The protocol will begin from the polishing of the detergent solubilized protein via size exclusion chromatography, along with considerations for use of detergents. SERCA is natively isolated from rabbit fasttwitch muscle (Møller \& Olesen, 2016).

[FIG 1 - SERCA ELUTION PROFILE]

The optimal starting point is a monodisperse peak in the size exclusion chromatogram, indicating a stable and homogenous sample (Figure 1). Assuming the sample is not intended for activity assays, locking the protein in a specific state using an inhibitor or other ligands is advisable.

\section{Equipment}

- AKTA Purifier HPLC system in a cold cabinet

- Superdex 200 Increase $10 / 300 \mathrm{GL}$ column

- DeNovix DS-11 spectrophotometer 
- SDS-PAGE equipment and $15 \%$ gels

- Optima MAX-XP Ultracentrifuge with a TLA-110 rotor

- Sartorius Vivaspin spin filter with a 100 kDa MW cut-off

\section{Buffers and Reagents}

- SERCA solubilized in DDM

- Size Exclusion buffer: $67 \mathrm{mM}$ MOPS (pH 6.8), $100 \mathrm{mM} \mathrm{KCl}, 10 \mathrm{mM} \mathrm{CaCl}, 5$ $\mathrm{mM} \mathrm{MgCl} 2,0.3 \mathrm{mg} / \mathrm{ml} \mathrm{DDM}$ (n-Dodecyl $\beta$-D-maltoside)

- SDS-PAGE loading buffer

\section{Procedure}

1. If needed, concentrate the sample to a volume suitable for size exclusion (50$500 \mu \mathrm{l})$, while keeping protein concentration low enough to avoid precipitation. If needed, split the sample into aliquots for multiple injections.

2. Pipette the sample into tubes compatible with ultracentrifugation and spin the sample at $50,000 \mathrm{rpm}$ at $4{ }^{\circ} \mathrm{C}$ for 35 minutes to sediment aggregates.

3. Inject the sample onto a column equilibrated with at least two column volumes of Size Exclusion buffer, and collect fractions ( $250-500 \mu \mathrm{L})$.

4. Run samples of the peak fractions on a $15 \%$ SDS-PAGE to check purity.

5. Collect pure peak fractions and concentrate to about $3 \mathrm{mg} / \mathrm{ml}$.

6. Use directly for Salipro reconstitution or flash freeze in liquid nitrogen and store at $-80^{\circ} \mathrm{C}$. 
Notes

1. Consider the choice and the cut-off of the spin filter. If a very low MW cut-off is chosen, not only is the protein concentrated, but also the detergent, due to the size of micelles.

\section{Reconstitution into Salipro}

[FIG 2 - SERCA-SALIPRO purification]

The gel-filtration profile of a reconstitution usually displays several peaks corresponding to the membrane protein incorporated into Salipro nanoparticles, peaks that originate from smaller lipid-only (empty) Salipro particles and monomeric, unbound saposin.

For further analysis, peak fractions are collected to isolate Salipro-SERCA complexes. In the case of SERCA, a careful collection of fractions might be required, due to the presence of two populations (monomers, dimers) of Salipro-SERCA. However, these are easily discriminated by particle size (Figure 2 ).

\section{Equipment}

- $\quad$ AKTA Purifier HPLC system

- Superdex 200 Increase $10 / 300 \mathrm{GL}$ column

- DeNovix DS-11 spectrophotometer

- Heat block

- Timer

\section{Buffers and Reagents}

- Purified SERCA at $2.9 \mathrm{mg} / \mathrm{ml}$ 
- Purified saposin at $1.2 \mathrm{mg} / \mathrm{ml}$

- Size Exclusion buffer: $67 \mathrm{mM}$ MOPS (pH 6.8), $100 \mathrm{mM} \mathrm{KCl}, 10 \mathrm{mM} \mathrm{CaCl}, 5$ $\mathrm{mM} \mathrm{MgCl} 2$

- Brain Lipid Extract (Sigma: B1502) stock at $20 \mathrm{mg} / \mathrm{ml}$ in $80 \mathrm{mM}$ Tris $\mathrm{pH} 7.5$, $1.5 \%(w / v)$ DDM

- Dilution buffer: $67 \mathrm{mM}$ MOPS (pH 6.8), $100 \mathrm{mM} \mathrm{KCl}, 10 \mathrm{mM} \mathrm{CaCl}, 5 \mathrm{mM}$ $\mathrm{MgCl}_{2}$

\section{Procedure}

1. Dilute brain lipid extract stock solution to a final concentration of $5 \mathrm{mg} / \mathrm{mL}$ using the dilution buffer, final detergent concentration of $0.375 \%(w / v)$.

2. Incubate $20 \mu \mathrm{L}$ of protein with $40 \mu \mathrm{L}$ of the $5 \mathrm{mg} / \mathrm{mL}$ BLE stock solution at room temperature $\left(20^{\circ} \mathrm{C}\right)$ for 10 minutes.

3. Add $360 \mathrm{uL}$ of $\operatorname{saposin} \mathrm{A}(1.2 \mathrm{mg} / \mathrm{mL})$ and incubate at room temperature for a further two minutes.

4. $500 \mathrm{uL}$ of dilution buffer is added.

5. The above reaction is set up five times, pooled and concentrated to $\sim 300 \mu \mathrm{L}$ for injection onto the HPLC column.

6. Inject the sample onto a column (superdex 200 10/300) equilibrated with at least two column volumes of Size Exclusion buffer, and collect fractions (100$200 u L)$.

7. Appropriate fractions are pooled and concentrated to the desired concentration or can be individually assessed using negative stain electron microscopy. 
1. When starting with an entirely new membrane protein target, it is recommended to perform small-scale screens on a Superdex S200 5/150 column first. Typically, three separate reconstitution mixes are set up and screened separately. Here, 1,3 of 5 ul of the membrane protein (concentration range $0.5-10 \mathrm{mg} / \mathrm{ml}$ ) are incubated with fixed volumes of Brain Lipids (5 ul at $5 \mathrm{mg} / \mathrm{ml}$ ) and Saposin (20 ul at $1.2 \mathrm{mg} / \mathrm{ml}$ ). After the addition of dilution buffer, the samples are subjected to a gel-filtration step, which allows to determine the optimal ratios for self-assembly of the Salipro particles.

2. Getting the Brain Lipid Extract stock solubilized might require alternating heating $\left(55^{\circ} \mathrm{C}\right)$, vortexing and bath sonication.

3. Other lipid mixtures can be used.

4. Screening for an optimum reconstitution protocol can be achieved using 2-10 $\mu \mathrm{L}$ of protein solution depending on the sensitivity of the detection of the chromatography system.

5. The throughput of the reconstitution protocol screening can be increased using automation and an autoloader.

6. An excess of saposin A is generally required for optimum reconstitution.

7. For the large scale preparation described above a number of reactions each with a final volume of $1 \mathrm{~mL}$ upon dilution (step4) are set up before pooling and concentrating with a spin filter of appropriate MW cut-off.

8. Adding $5-10 \%$ glycerol to the size exclusion buffer may further stabilize the membrane protein, especially when freezing/thawing, however the presence 
of glycerol might hamper the contrast in future cryo-EM studies for highresolution.

9. For larger membrane protein complexes, different column materials (e.g. Superose 6) may prove beneficial.

\section{Electron Microscopy of Salipro Particles}

Here we describe how to image the SERCA-Salipro particles using negative stain EM and how to reconstruct a 3D volume from the data. Compared to cryo-EM, negative stain EM has a very limited maximum resolution, revealing only the overall shape of imaged particles. However, sample preparation is quick and the contrast is high, making it well suited for screening samples before moving to high-resolution cryoEM.

\section{Equipment}

- PELCO EasiGlow Glow Discharge Cleaning System

- Tecnai G2 Spirit electron microscope equipped with an LaB6 filament and operated at an acceleration voltage of $120 \mathrm{kV}$ and a Tietz F415 CCD camera with $4 \mathrm{k} \times 4 \mathrm{k}$ pixels

- Leginon data acquisition software (Suloway et al., 2005)

- Appion data processing suite (Lander et al., 2009)

- EMAN software ((Ludtke, Baldwin, \& Chiu, 1999)

- RELION version 2.0 (Scheres, 2012)

- Chimera software (Pettersen et al., 2004) 
- Computing resources - here we used a GPU cluster (Dell PowerEdge C4130 w/ 2 x Xeon E5-2620v4, 128 GB RAM, 4 x NVIDIA TESLA K80)

\section{Buffers and Reagents}

- Sample dilution buffer: $67 \mathrm{mM}$ MOPS (pH 6.8), $100 \mathrm{mM} \mathrm{KCl}, 5 \mathrm{mM} \mathrm{MgCl}, 4$ mM EGTA, $0.5 \mathrm{mM}$ BeSO4, $10 \mathrm{mM} \mathrm{NaF}$

- SERCA1a-Salipro sample at $0.24 \mathrm{mg} / \mathrm{mL}$

- Commercial copper G400-C3 grids overlaid with carbon, glow discharged at $25 \mathrm{~mA}$ for $45 \mathrm{~s}$ prior to use.

- $\quad 2 \%(w / v)$ uranyl formate (Ohi, Li, Cheng, \& Walz, 2004)

\section{Procedure}

1. SERCA1a-Salipro complex is diluted six-fold using the sample dilution buffer to $\sim 40 \mathrm{ug} / \mathrm{mL}$ in the presence of the conformation stabilizing (E2P) inhibitor BeFx (from BeSO4 and $\mathrm{NaF}$ ) and incubated on ice for $1 \mathrm{~h}$.

2. $3 \mathrm{uL}$ of protein solution was added to a freshly glow discharged grid.

3. After $\sim 10$ seconds, the excess protein solution was removed by blotting the edge of the grid against a piece of Whatman grade 4 filter paper.

4. $3 \mathrm{uL}$ of $2 \%(\mathrm{w} / \mathrm{v})$ uranyl formate stain was immediately added and excess stain was removed by blotting the same edge of the grid as before against the filter paper.

5. Staining was repeated twice more as per step 3.

6. The grid was left to air dry for a few minutes before use.

7. Images were collected on a Tecnai G2 Spirit microscope using the automated data collection software, Leginon. 
8. Particles were picked automatically using dogpicker.py as implemented in Appion, and converted to boxfiles in EMAN.

9. Particles were extracted in Relion 2.0 and submitted to subsequent rounds of 2D and 3D classification (Figure 3).

[FIG 3 - Micrograph and class averages]

10. 3D volumes were manually inspected in Chimera and a crystal-derived model of SERCA1a was manually fitted into the volumes (Figure 4).

[FIG 4 - 3D SERCA-SALIPRO]

Notes

1. If aggregates are observed in the micrographs, subjecting the sample to ultracentrifugation prior to preparing grids might be used to remove them.

2. Protein concentration of the sample applied to the grid will have to be screened to obtain micrographs containing as many particles as possible, while still having them isolated from one another.

3. Data processing can be greatly enhanced and sped up using a GPU enabled workstation or cluster (Kimanius, Forsberg, Scheres, \& Lindahl, 2016).

\section{Summary and Conclusion}

Reconstitution of membrane proteins into Salipro nanoparticles follows a fairly straightforward protocol, and optimizations of e.g. lipid type, incubation time and temperature, and protein:saposin:lipid ratios can be screened on a small scale. For large-scale production, several methods can be used. One option is to perform 
several runs of the same reconstitution with subsequent purification on a mediumsized size-exclusion columns $(24 \mathrm{ml})$, and then pooling the relevant peak fractions from each SEC run and concentrating, as described earlier (Frauenfeld et al, 2016). Another approach is presented here, where several reconstitution mixtures are pooled and concentrated prior to purification on a medium-sized size-exclusion column.

Alternatively, it is possible to perform the reconstitution with higher amounts and volumes (up to $5 \mathrm{ml}$ ) and inject the mixture on a size-exclusion column with larger volume $(120 \mathrm{ml})$. However this large-column approach should only be performed if reconstitution conditions are well established on the small- and medium scale.

Preparing grids with Salipro samples and collecting data can be performed on the same day as reconstitution, especially with the help of data collection software such as Appion. Nevertheless, for SERCA-Salipro we could also flash-freeze samples and thaw later for grid preparation without significant aggregation observed in the micrographs.

Given the small size of the particle imaged here, and with the use of a GPU-cluster to accelerate processing, we were able to go from micrographs to 3D volume in a few days. The generated 3D volume fits the crystallographic model very well, and it is worth noting that SERCA at $110 \mathrm{kDa}$ is still on the small side of what can be characterized to high resolution using cryo-EM.

We have found these procedures to work well for a range of P-type ATPases and other transporters, and are currently exploring the possibilities for also extracting Salipro particles directly from native membranes. There are also interesting applications to small-angle scattering studies, and expressed in E. coli, saposin can be 
subjected to deuteration for neutron scattering studies. It remains to be seen if Salipro complexes of membrane proteins can be crystallized, but given the rather adaptive and dynamic nature of the Salipro interaction it would not seem unfeasible.

\section{References}

Ahn, V. E., Leyko, P., Alattia, J.-R., Chen, L., \& Privé, G. G. (2006). Crystal structures of saposins A and C. Protein Science : A Publication of the Protein Society, 15(8), 1849-57. http://doi.org/10.1110/ps.062256606

Baker, M. R., Fan, G., \& Serysheva, I. I. (2015). Single-particle cryo-EM of the ryanodine receptor channel in an aqueous environment. European Journal of Translational Myology, 25(1), 35-48. http://doi.org/10.4081/ejtm.2015.4803

Bayburt, T. H., Grinkova, Y. V., \& Sligar, S. G. (2002). Self-Assembly of Discoidal Phospholipid Bilayer Nanoparticles with Membrane Scaffold Proteins. Nano Letters, 2(8), 853-856. http://doi.org/10.1021/nl025623k

Caffrey, M. (2015). A comprehensive review of the lipid cubic phase or in meso method for crystallizing membrane and soluble proteins and complexes. Acta Crystallographica. Section F, Structural Biology Communications, 71(Pt 1), 3-18. http://doi.org/10.1107/S2053230X14026843

Danev, R., \& Baumeister, W. (2016). Cryo-EM single particle analysis with the Volta phase plate. eLife, 5(MARCH2016), 1-14. http://doi.org/10.7554/eLife.13046

Faham, S., \& Bowie, J. U. (2002). Bicelle crystallization: a new method for crystallizing membrane proteins yields a monomeric bacteriorhodopsin 
structure. Journal of Molecular Biology, 316(1), 1-6.

http://doi.org/10.1006/jmbi.2001.5295

Frauenfeld, J., Gumbart, J., Sluis, E. O. van der, Funes, S., Gartmann, M., Beatrix, B., ... Beckmann, R. (2011). Cryo-EM structure of the ribosome-SecYE complex in the membrane environment. Nature Structural \& Molecular Biology, 18(5), 614-21. http://doi.org/10.1038/nsmb.2026

Frauenfeld, J., Löving, R., Armache, J.-P., Sonnen, A. F.-P., Guettou, F., Moberg, P., ... Nordlund, P. (2016). A saposin-lipoprotein nanoparticle system for membrane proteins. Nature Methods, 13(4), 345-51. http://doi.org/10.1038/nmeth.3801

Gao, Y., Cao, E., Julius, D., \& Cheng, Y. (2016). TRPV1 structures in nanodiscs reveal mechanisms of ligand and lipid action. Nature, 534(7607), 347-51. http://doi.org/10.1038/nature17964

Gourdon, P., Andersen, J. L., Hein, K. L., Bublitz, M., Pedersen, B. P., Liu, X.-Y., ... Morth, J. P. (2011). HiLiDe-Systematic Approach to Membrane Protein Crystallization in Lipid and Detergent. Crystal Growth \& Design, 11(6), 20982106. http://doi.org/10.1021/cg101360d

Jamshad, M., Lin, Y.-P., Knowles, T. J., Parslow, R. A., Harris, C., Wheatley, M., ... Dafforn, T. R. (2011). Surfactant-free purification of membrane proteins with intact native membrane environment. Biochemical Society Transactions, 39(3), 813-8. http://doi.org/10.1042/BST0390813

Kimanius, D., Forsberg, B. O., Scheres, S. H., \& Lindahl, E. (2016). Accelerated cryo-EM structure determination with parallelisation using GPUs in RELION-2. eLife, 5, 1-21. http://doi.org/10.7554/eLife.18722

Kühlbrandt, W. (2014). Biochemistry. The resolution revolution. Science (New 
York, N.Y.), 343(6178), 1443-4. http://doi.org/10.1126/science.1251652

Lander, G. C., Stagg, S. M., Voss, N. R., Cheng, A., Fellmann, D., Pulokas, J., ... Carragher, B. (2009). Appion: an integrated, database-driven pipeline to facilitate EM image processing. Journal of Structural Biology, 166(1), 95-102. http://doi.org/10.1016/j.jsb.2009.01.002

Loll, P. J. (2014). Membrane proteins, detergents and crystals: what is the state of the art? Acta Crystallographica. Section F, Structural Biology Communications, 70(Pt 12), 1576-83. http://doi.org/10.1107/S2053230X14025035

Ludtke, S. J., Baldwin, P. R., \& Chiu, W. (1999). EMAN: semiautomated software for high-resolution single-particle reconstructions. Journal of Structural Biology, 128(1), 82-97. http://doi.org/10.1006/jsbi.1999.4174

Lyons, J. A., Shahsavar, A., Paulsen, P. A., Pedersen, B. P., \& Nissen, P. (2016). Expression strategies for structural studies of eukaryotic membrane proteins. Current Opinion in Structural Biology, 38, 137-44. http://doi.org/10.1016/j.sbi.2016.06.011

Møller, J. V, \& Olesen, C. (2016). Preparation of Ca2+-ATPase1a Enzyme from Rabbit Sarcoplasmic Reticulum. In M. Bublitz (Ed.), P-Type ATPases: Methods and Protocols (pp. 11-17). New York, NY: Springer New York. http://doi.org/10.1007/978-1-4939-3179-8_3

Ohi, M., Li, Y., Cheng, Y., \& Walz, T. (2004). Negative Staining and Image Classification - Powerful Tools in Modern Electron Microscopy. Biological Procedures Online, 6(1), 23-34. http://doi.org/10.1251/bpo70

Pettersen, E. F., Goddard, T. D., Huang, C. C., Couch, G. S., Greenblatt, D. M., Meng, E. C., \& Ferrin, T. E. (2004). UCSF Chimera--a visualization system for 
exploratory research and analysis. Journal of Computational Chemistry, 25(13), 1605-12. http://doi.org/10.1002/jcc.20084

Popovic, K., Holyoake, J., Pomès, R., \& Privé, G. G. (2012). Structure of saposin A lipoprotein discs. Proceedings of the National Academy of Sciences of the United States of America, 109(8), 2908-12.

http://doi.org/10.1073/pnas.1115743109

Postis, V., Rawson, S., Mitchell, J. K., Lee, S. C., Parslow, R. A., Dafforn, T. R., ... Muench, S. P. (2015). The use of SMALPs as a novel membrane protein scaffold for structure study by negative stain electron microscopy. Biochimica et Biophysica Acta, 1848(2), 496-501. http://doi.org/10.1016/j.bbamem.2014.10.018

Poulos, S., Morgan, J. L. W., Zimmer, J., \& Faham, S. (2015). Bicelles coming of age: an empirical approach to bicelle crystallization. Methods in Enzymology, 557, 393-416. http://doi.org/10.1016/bs.mie.2014.12.024

Scheres, S. H. W. (2012). RELION: implementation of a Bayesian approach to cryo-EM structure determination. Journal of Structural Biology, 180(3), 51930. http://doi.org/10.1016/j.jsb.2012.09.006

Suloway, C., Pulokas, J., Fellmann, D., Cheng, A., Guerra, F., Quispe, J., ... Carragher, B. (2005). Automated molecular microscopy: the new Leginon system. Journal of Structural Biology, 151(1), 41-60. http://doi.org/10.1016/j.jsb.2005.03.010 


\section{Acknowledgements}

This work was in part funded through the Horizon 2020 programme of the European Union (iNEXT grant No. 653706). Molecular graphics and analyses were performed with the UCSF Chimera package. Chimera is developed by the Resource for Biocomputing, Visualization, and Informatics at the University of California, San Francisco (supported by NIGMS P41-GM103311).

\section{Figure Legends}

\section{Figure 1}

Elution profile from size exclusion chromatography of SERCA solubilized in DDM. Peak 1 is at the void volume and was discarded, as was peak 2 which might correspond to SERCA dimers. Peak 3 contains purified SERCA monomer and was collected for SERCA-Salipro reconstitution.

\section{Figure 2}

Elution profile from size exclusion chromatography (SEC) of SERCA-Salipro reconstitution. Peak 2 contains the SERCA monomers used for negative stain cryoEM. Peak 1 likely contains SERCA dimers reconstituted in Salipro, but with careful fractionation a sample of almost pure monomeric form could be obtained, as evident from the micrographs and class averages (Figure 3). Peak three is composed of lipid-only (empty) Salipro particles. Typically, a peak corresponding to free saposin A is evident at $\sim 17.5 \mathrm{~mL}$, however concentration of the sample using a $100 \mathrm{KDa}$ MWCO spin filter prior to injection to SEC can decrease the concentration of saposin 
A and possibly the empty salipro nanoparticles with respect to the protein of interest.

\section{Figure 3}

a) Micrograph of the negative stain SERCA-Salipro sample. The micrograph is cropped to cover a quarter of the collected micrograph. The scale bar represents 100 $\mathrm{nm}$. The expected shape of Salipro discs with the cytosolic domains of SERCA extending out on one side is clearly visible in most of the particles. Little to no aggregation was observed in the micrographs. b) Selected 2D class averages as output from a 2D classification in Relion. Sample are in a 72 pixel box with 3.15

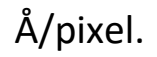

\section{Figure 4}

The 3D envelope as calculated in RELION with a crystallographic model of SERCA (PDB: 3B9B) manually docked into the volume in Chimera. Three side views, 90 degrees apart, along with views from the top and bottom. The transmembrane domain of SERCA is colored in tan, the actuator $(A)$ domain in yellow, the nucleotidebinding $(\mathrm{N})$ domain in red, and the phosphorylation $(\mathrm{P})$ domain in blue. 
Figure 1

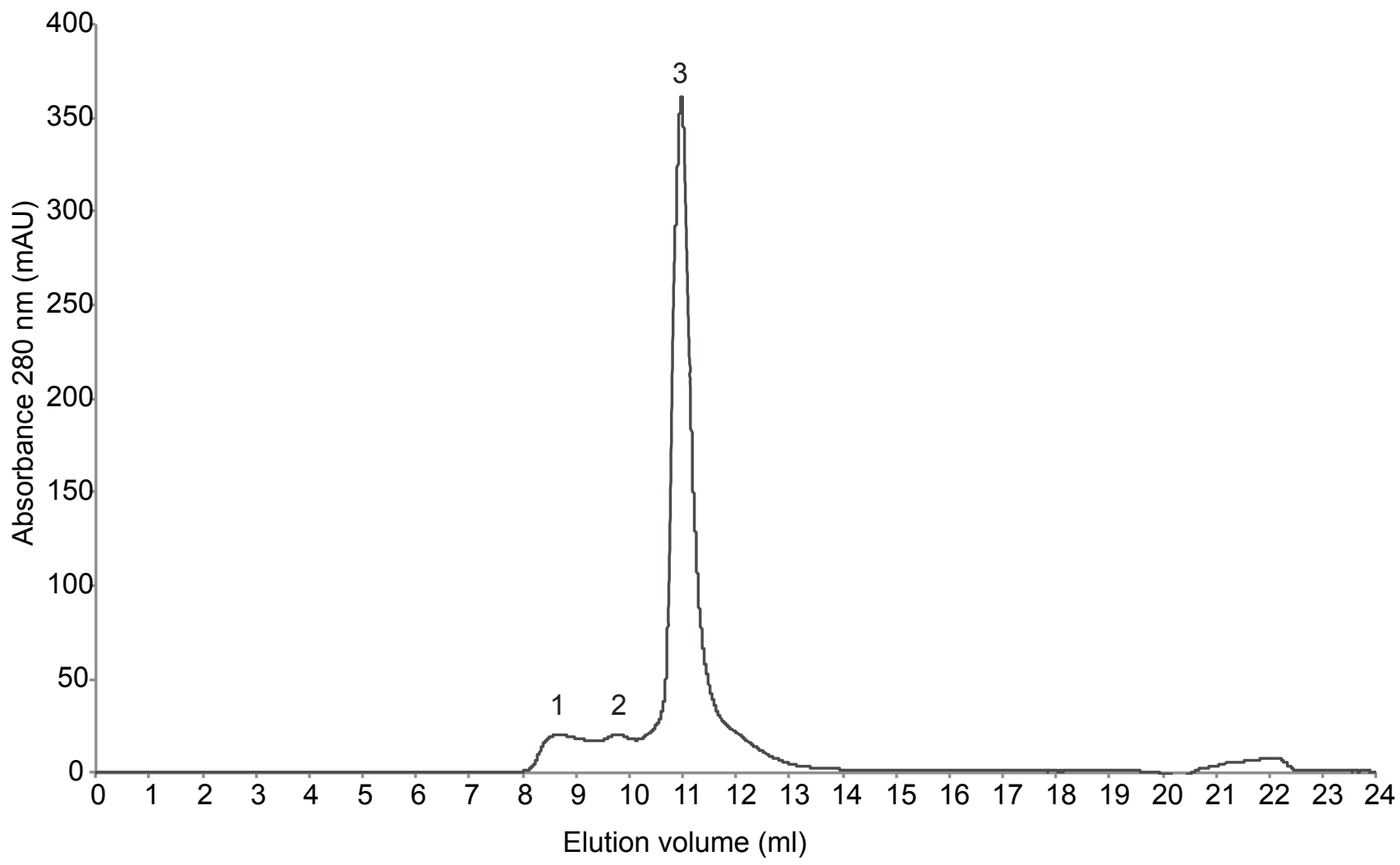


Figure 2

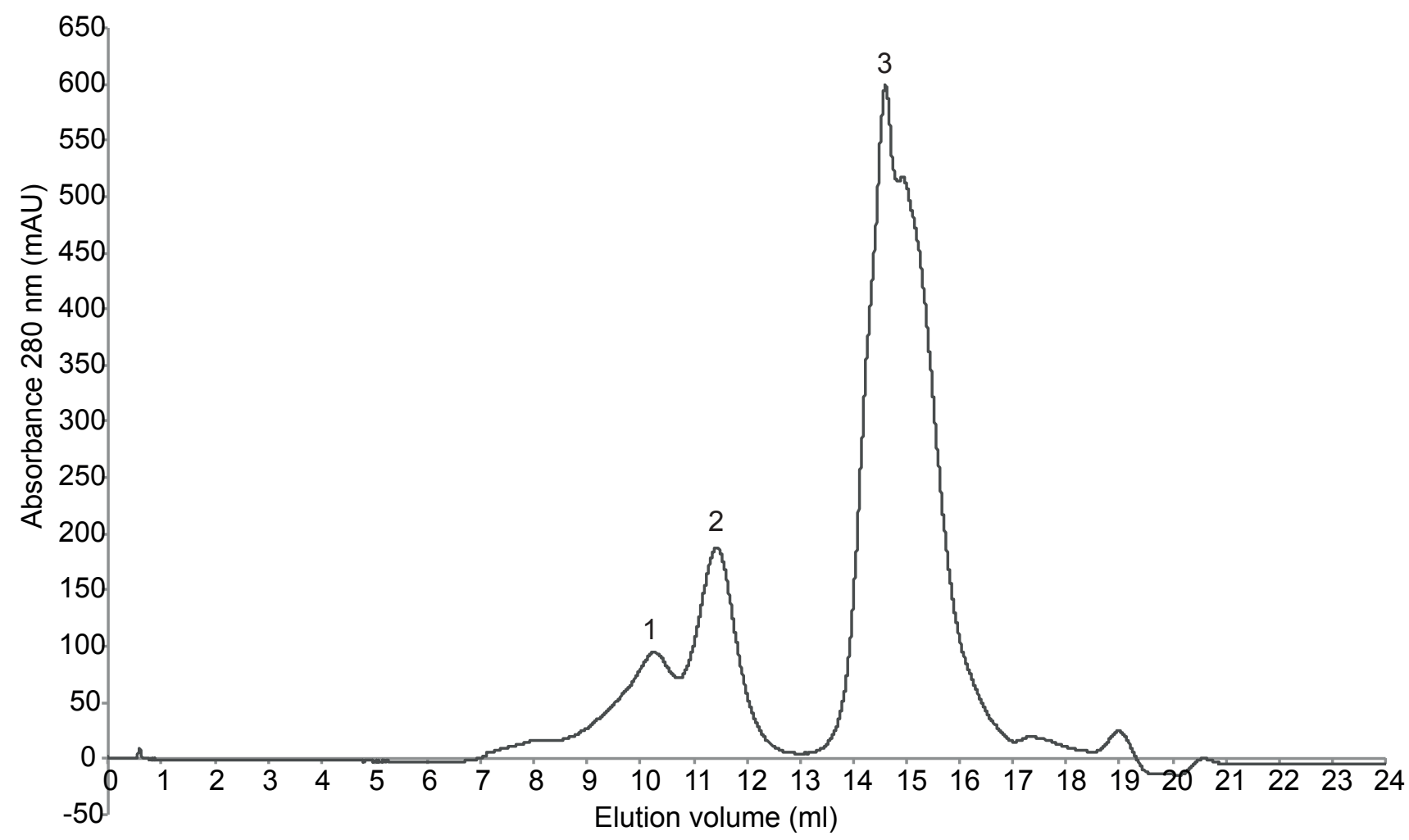


Figure 3

a

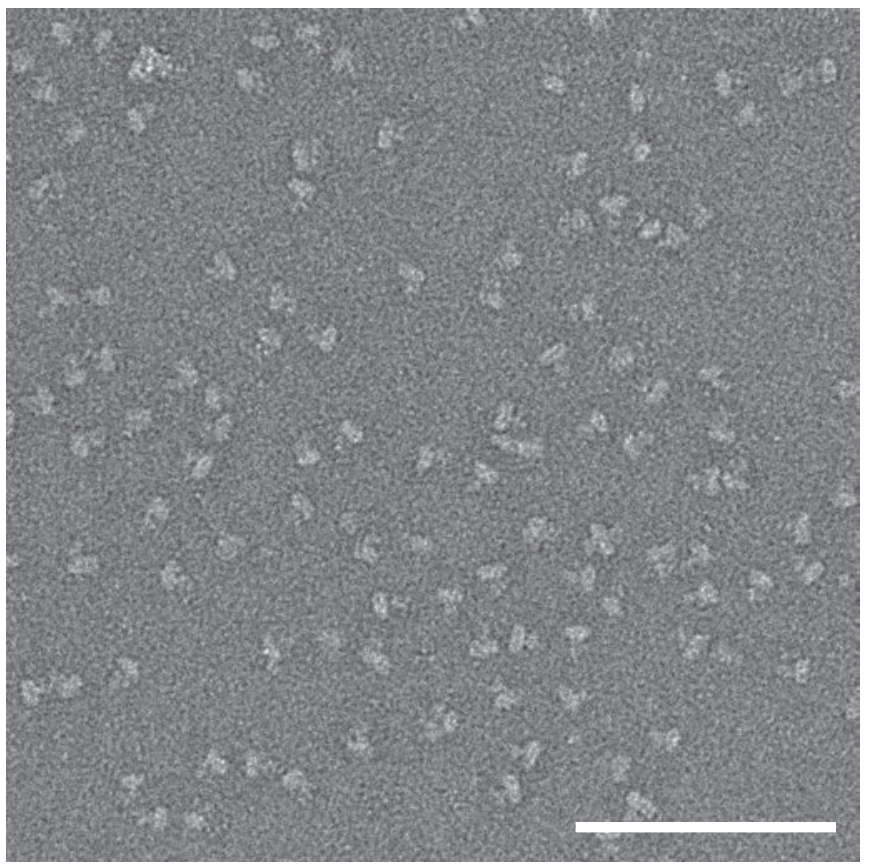

b
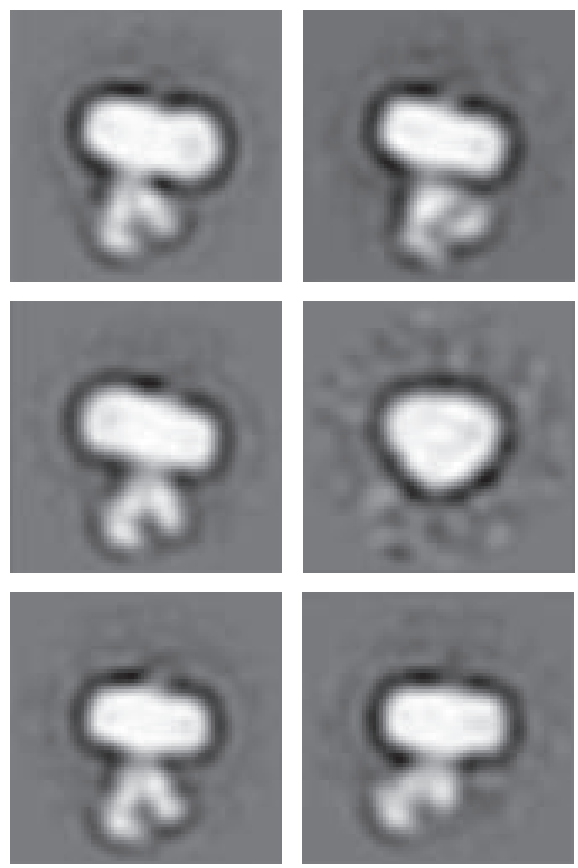
Figure 4
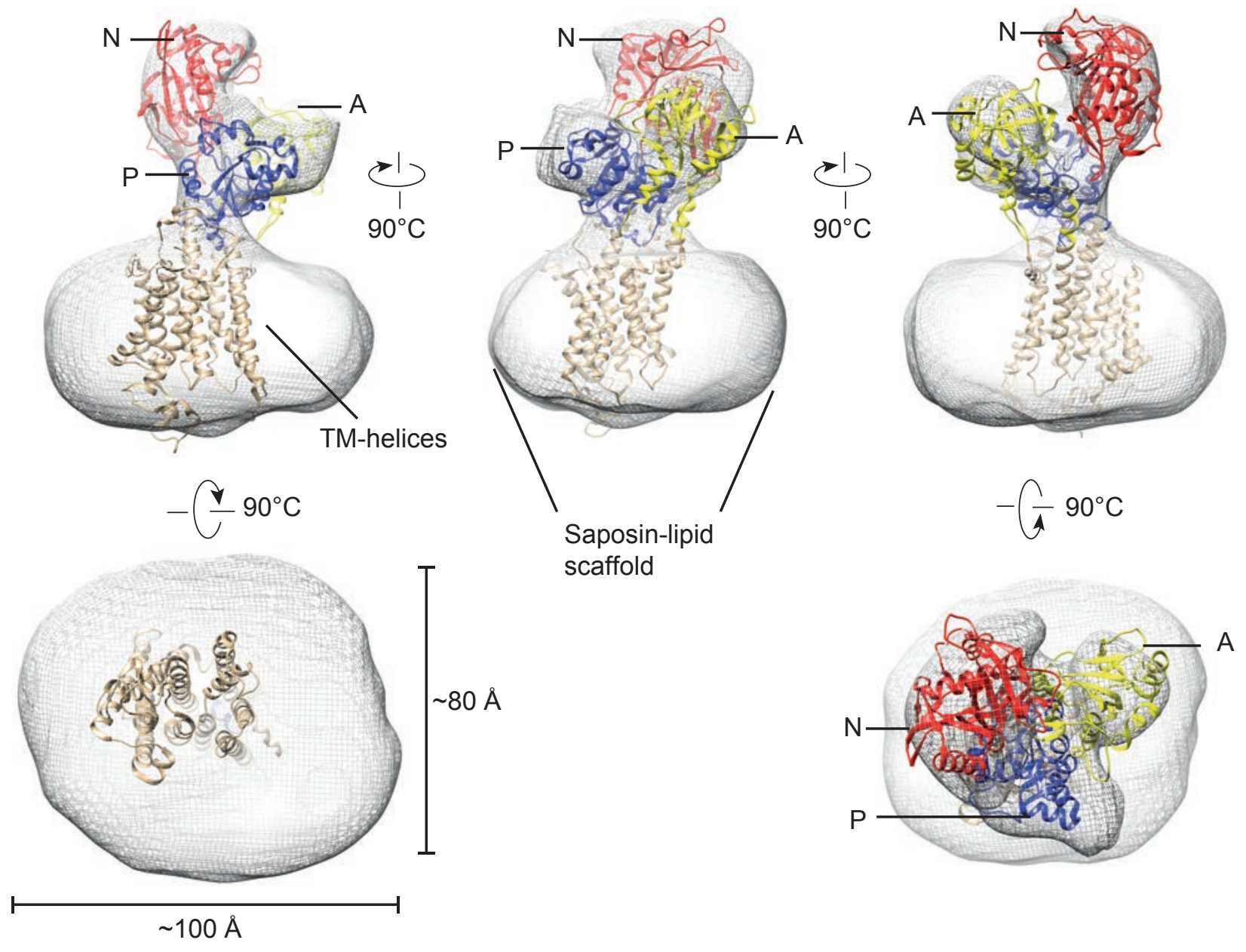